\section{Commentary: We don't need no postop AF... All in all it's just another $(\beta)$-block in the wall}

\author{
Rupak Mukherjee, $\mathrm{PhD}$
}

Atrial fibrillation (AF) can complicate the treatment and prognoses of a number of conditions. Despite advances in surgical techniques, prophylaxis, and refinements of antiarrhythmic algorithms, supraventricular arrhythmias, of which $\mathrm{AF}$ is one of the most prominent arrhythmia, remain a common postoperative complication of cardiac surgery. ${ }^{1,2}$ Postoperative AF (PoAF) increases the risk of stroke, myocardial infarction, heart failure, costs, and prolongs length of stay. ${ }^{2}$ The incidence of PoAF has been reported to vary between $10 \%$ and $60 \%$ depending on the type of cardiac surgery and the underlying myocardial substrate. ${ }^{2}$ Mechanisms that promulgate the development of PoAF remain elusive but likely involve a combination of heightened release of inflammatory factors, increased oxidative stress, changes in processes that alter physiological excitation-contraction coupling, and neurohormonal activation during and following cardiac surgery (Figure 1). ${ }^{2}$

There have been a number of studies and randomized controlled trials that have examined the efficacy of various interventions on the risk of supraventricular arrhythmias, including PoAF. ${ }^{1,3-5}$ A Cochrane systematic review published in 2013, updating the original results from 2004, performed a meta-analysis on 118 trials that included more than 17,000 subjects. ${ }^{1}$ There were multiple interventions studied including amiodarone, $\beta$-blockers, sotalol, magnesium, atrial pacing, and posterior pericardiotomy. Each of the studied interventions significantly

From the Division of Cardiothoracic Surgery, Department of Surgery, Medical University of South Carolina, Charleston, SC.

Disclosures: The author reported no conflicts of interest.

The Journal policy requires editors and reviewers to disclose conflicts of interest and to decline handling or reviewing manuscripts for which they may have a conflict of interest. The editors and reviewers of this article have no conflicts of interest.

Received for publication July 24, 2020; revisions received July 24, 2020; accepted for publication Aug 4, 2020; available ahead of print Sept 2, 2020.

Address for reprints: Rupak Mukherjee, PhD, Division of Cardiothoracic Surgery Research, 30 Courtenay Dr, Suite 338, Charleston, SC 29425 (E-mail: mukherr@musc.edu).

JTCVS Open 2020;3:88-90

2666-2736

Copyright $(2020$ The Authors. Published by Elsevier Inc. on behalf of The American Association for Thoracic Surgery. This is an open access article under the CC BY-NCND license (http://creativecommons.org/licenses/by-nc-nd/4.0/).

https://doi.org/10.1016/j.xjon.2020.08.003

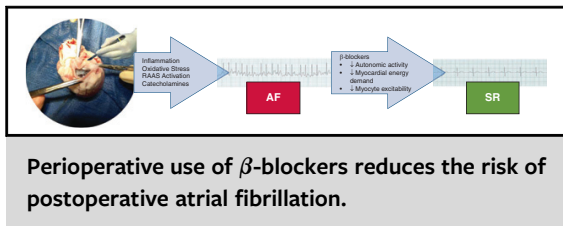

CENTRAL MESSAGE

This is a commentary on a metaanalysis performed to examine the effects of using $\beta$-blockers on the development of atrial fibrillation in cardiac patients.

reduced the rate of PoAF after cardiac surgery compared with controls. Among the pharmacologic interventions, $\beta$ blockers and sotalol appeared to provide better efficacy in reducing PoAF compared to magnesium and amiodarone. ${ }^{1}$ Since the publication of the Cochrane systematic review, other meta-analyses have reported on the effects of colchicine and vitamin $\mathrm{C}$ on PoAF. ${ }^{3-5}$ As secondary outcomes, these interventions, in general, were shown to decrease length of hospital stay and decrease hospital costs. ${ }^{1,3-5}$

In this issue of JTCVS Open, Masuda and colleagues ${ }^{6}$ present the results of a meta-analyses in which the investigators selected studies that examined the effects of $\beta$ blockers on "isolated" PoAF. To select studies for this meta-analysis, the authors defined "isolated" AF as AF being the sole arrhythmia examined. In addition, the selected studies solely used $\beta$-blockers (also excluding sotalol) administered perioperatively as treatment for PoAF. Based on these criteria, the authors included 17 randomized controlled trials in this meta-analysis. To account for potential bias, the authors assigned a score based on the Jadad scale $^{7}$ to each study based on randomization of patients, blinding of treatment groups, and whether the studies accounted for all included subjects. The overall conclusion of the meta-analysis was that $\beta$-blockers reduced the risk for isolated PoAF (odds ratio of 0.52) compared with the control arms. Somewhat counterintuitively, subgroup analysis revealed that the risk for PoAF with perioperative $\beta$ blockade was lowest in patients who underwent on pump coronary artery bypass grafting compared with off-pump coronary artery bypass grafting or coronary artery bypass grafting combined with valve procedures. Moreover, postoperative administration of $\beta$-blockers appeared to be more efficacious in reducing PoAF risk than $\beta$-blockers administered before or during the procedure. Finally, 


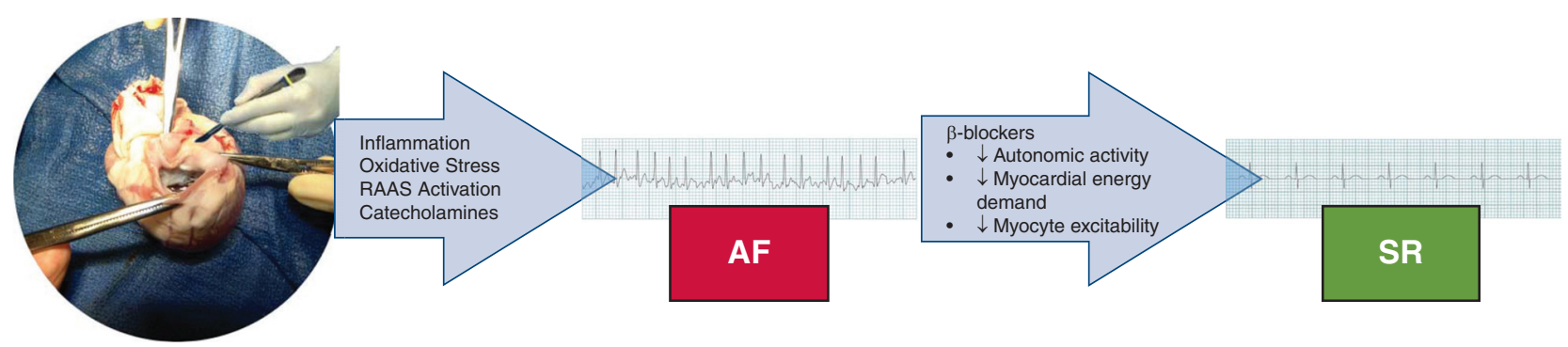

FIGURE 1. Following cardiac surgery, there is an increase in inflammatory cytokines, oxidative stress, activation of the RAAS, and release of catecholamines. Each of these factors, alone or in combination, may serve as triggers for increased automaticity or electrical conduction abnormalities within the atrium generating AF in some patients. The perioperative use of $\beta$-blockers, which could attenuate the chronotropic and inotropic effects of the catecholamine surge, can reduce the development of AF, potentially allowing SR in these patients. RAAS, Renin-angiotensin-aldosterone system; $A F$, atrial fibrillation; $S R$, sinus rhythm. Source of ECG strips: Life in the Fast Lane ECG Library (https://litfl.com/ecg-library/).

intravenous administration of landiolol, an ultra-short "super-selective" $\beta 1$-blocker, appeared to confer the lowest risk of PoAF development compared with other $\beta$-blockers.

It is no surprise that $\beta$-blockers have the potential to reduce PoAF development. $\beta$-blockers can attenuate the effects of the catecholamine surge that can occur following cardiac surgery by antagonism of chronotropic and inotropic pathways (Figure 1). Moreover, $\beta$-blockers may reduce the incidence of triggered electrical activity of myocytes secondary to slowing of the heart rate that reduces myocardial energy demand and allows for better perfusion of the myocardium during diastole. Based on these effects of $\beta$-blockers on PoAF, perioperative treatment with $\beta$-blockers is recommended as a Class 1A indication for patients undergoing cardiac surgery. ${ }^{8}$

The results from the meta-analysis presented by Masuda and colleagues ${ }^{6}$ are in overall agreement with the conclusions of the Cochrane systematic review that included many more studies on arrhythmias after cardiac surgery. ${ }^{1}$ While the meta-analyses by Masuda and colleagues do state that the use of $\beta$-blockers approximately halved the risk of developing PoAF after cardiac surgery, and the $95 \%$ confidence interval for the reduced risk of PoAF with $\beta$-blockers is reported to range between 0.41 and 0.66 (does not include 1 ), the authors report that the $P$ value for the predication interval was statistically not significant $(P=.31)$. Many of the other subgroup comparisons made between the $\beta$-blocker group and control group also failed to attain statistical significance (defined as $P$ values of less than .05). The authors are generally silent on potential reasons for the results of the analyses not achieving statistical significance.

What is the "take-home" message for cardiac surgeons from this study? The authors, in the conclusion, suggest that $\beta$-blockers be used in patients undergoing on-pump coronary artery bypass procedures and that intravenous landiolol may be better in reducing the risk of developing PoAF. That being said, the results from this meta-analyses do not provide any additional information with respect to modifications of guidelines.
Given the complications that PoAF imposes on management of patients following cardiac surgery, there remains the need to continue research and development of strategies to reduce/eliminate PoAF. There are many factors that contribute to restrict research in this field. First, cardiac surgery procedures result in the emergence of multiple factors, including oxidative stress and activation of various neurohormonal pathways that, either alone or in combination, can increase automaticity and may trigger electrical activation within the atria. ${ }^{2}$ Isolation of these pathways to determine the contribution of each can be difficult. Indeed, this may be the very reason why antiarrhythmics (like amiodarone or sotalol), antioxidants (like vitamin $\mathrm{C}$ ), and colchicine, in addition to $\beta$-blockers, have all been shown to be efficacious in reducing PoAF development following cardiac surgery. ${ }^{1-3,5}$ Second, it has been difficult to generate animal models that recapitulate the pathophysiology of human AF (for an excellent recent review, see Schüttle and colleagues $^{9}$ ). Even after occlusion of components of the coronary circulation to induce myocardial infarction and/ or myocardial ischemia-reperfusion, very few-if anystudies report $\mathrm{AF}$ development in species ranging from mice to pigs. Therefore, examination of cellular and molecular processes that may contribute to $\mathrm{AF}$, particularly PoAF, has been problematic. Finally, isolated cell systems do not currently provide an adequate substrate to investigate mechanisms of triggered electrical activation in a setting that mimics the postoperative milieu. Taken together, the best means to study effective treatment(s) to reduce and/or eliminate the development of PoAF following cardiac surgery remains randomized controlled trials and meta-analyses, such as the one presented here by Masuda and colleagues, ${ }^{6}$ allows the formulation and refinement of clinical guidelines for this insidious arrhythmia.

\section{References}

1. Arsenault KA, Yusuf AM, Crystal E, Healey JS, Morillo CA, Nair GM, et al. Interventions for preventing post-operative atrial fibrillation in patients undergoing heart surgery. Cochrane Database Syst Rev. 2013;CD003611. 
2. Turagam MK, Downey FX, Kress DC, Sra J, Tajik AJ, Jahangir A. Pharmacological strategies for prevention of postoperative atrial fibrillation. Expert Rev Clin Pharmacol. 2015;8:233-50.

3. Hu X, Yuan L, Wang H, Li C, Cai J, Hu Y, et al. Efficacy and safety of vitamin c for atrial fibrillation after cardiac surgery: a meta-analysis with trial sequential analysis of randomized controlled trials. Int J Surg. 2017;37:58-64.

4. Lee JZ, Singh N, Howe CL, Low SW, Huang JJ, Ortega G, et al. Colchicine for prevention of post-operative atrial fibrillation: a meta-analysis. JACC Clin Electrophysiol. 2016;2:78-85.

5. Lennerz C, Barman M, Tantawy M, Sopher M, Whittaker P. Colchicine for primary prevention of atrial fibrillation after open-heart surgery: systematic review and meta-analysis. Int J Cardiol. 2017;249:127-37.

6. Masuda Y, Luo HD, Kang GS, Teoh L-KK, Kofidis T. Meta-analysis of the benefit of beta-blockers for the reduction of isolated atrial fibrillation incidence after cardiac surgery. J Thorac Cardiovasc Surg Open. 2020;3: 66-85.

7. Jadad AR, Moore RA, Carroll D, Jenkinson C, Reynolds DJ, Gavaghan DJ, et al. Assessing the quality of reports of randomized clinical trials: is blinding necessary? Control Clin Trials. 1996;17:1-12.

8. January CT, Wann LS, Calkins H, Chen LY, Cigarroa JE, Cleveland JC Jr, et al. 2019 AHA/ACC/HRS focused update of the 2014 AHA/ACC/HRS guideline for the management of patients with atrial fibrillation: a report of the American College of Cardiology/American Heart Association task force on clinical practice guidelines and the Heart Rhythm Society in collaboration with the Society of Thoracic Surgeons. Circulation. 2019; 140:e125-51.

9. Schüttler D, Bapat A, Kääb S, Lee K, Tomsits P, Clauss S, et al. Animal models of atrial fibrillation. Circ Res. 2020;127:91-110. 